\title{
Effect of Tai Chi Quan on the Pressure Pain Thresholds of Lower Back Muscles in Healthy Women
}

\author{
Rui Wang', Xiao-Long Chang' ${ }^{2}$ Suparata Kiartivich', Xue-Qiang Wang $\mathbb{D}^{1,3}$ \\ 'Department of Sport Rehabilitation, Shanghai University of Sport, Shanghai, People's Republic of China; ${ }^{2}$ School of Physical Education and Training, \\ Shanghai University of Sport, Shanghai, People's Republic of China; ${ }^{3}$ Department of Rehabilitation Medicine, Shanghai Shangti Orthopaedic Hospital, \\ Shanghai, People's Republic of China
}

Correspondence: Xue-Qiang Wang, Department of Sport Rehabilitation, Shanghai University of Sport, 399 Changhai Road, Shanghai, 200438, People's Republic of China, Tel +86 I86 016 I 2741, Email wangxueqiang@sus.edu.cn

Purpose: Although studies on the improvement of pain after exercise are increasingly diverse, whether Tai Chi Quan can improve the pressure pain thresholds remains unknown. This study was to observe the effect of Tai Chi Quan on the pressure pain thresholds of lower back muscles in healthy women.

Patients and Methods: This was a randomized controlled trial. Sixty healthy women aged $18-40$ years were randomly assigned to Tai Chi group or control group. The Tai Chi group received 40-minute practice, and the control group received 5-minute sham Tai Chi Quan practice and 35-minute rest. The pressure pain thresholds of the longissimus thoracis, iliocostalis lumborum, multifidus muscle, quadratus lumborum, gluteus medius and supraspinous ligament were assessed before and immediately after intervention.

Results: The pressure pain thresholds of test points in the Tai Chi group showed substantial improvements after exercise, whereas those in the control group did not improve. Overall, the pressure pain thresholds in the Tai Chi group significantly increased compared with the control group (longissimus thoracis: $p=0.000$, iliocostalis lumborum: $p=0.000$, multifidus muscle: $p=0.000$, quadratus lumborum: $\mathrm{p}=0.012$, gluteus medius: $\mathrm{p}=0.000$ and supraspinous ligament: $\mathrm{p}=0.000$ ).

Conclusion: Forty minutes of Tai Chi Quan exercise remarkably increased the pressure pain thresholds of lower back muscles in healthy women, and thresholds at by an these points increased average of $17.2 \%$.

Keywords: complementary and alternative medicine, pain thresholds, chronic pain, rehabilitation

\section{Introduction}

Pressure pain thresholds (PPTs) and tenderness points are predictors of chronic pain and can be measured using a digital pain manometer. ${ }^{1}$ The increase in individual pain threshold is correlated with decreased pain, and the decrease in muscle stiffness is closely related to muscle relaxation and pain relief., ${ }^{2,3}$ Peak pressure may be relevant for pain onset, and various tissue properties have a different impact on PPTs. ${ }^{4}$ PPTs also vary amongst people with different genders and weights. ${ }^{5}$ Healthy females have lower PPTs in the gluteus and tensor fascia than healthy males. ${ }^{4}$ Obese people have higher PPTs than people with normal weight. ${ }^{6}$

A meta-analysis reported that individuals with chronic pain have considerably lower PPTs compared with healthy people. ${ }^{7}$ Low back pain (LBP) is one of the most common chronic pain conditions and a major public health problem worldwide with high prevalence and high disability rate, which contribute huge medical burden and the loss of personal productivity. ${ }^{89}$ Females with LBP have substantially lower PPTs at the fifth lumbar level, gluteus maximus and gluteus medius than males with LBP. ${ }^{10,11}$ Peter et al reported that patients with chronic LBP have lower pressure sensitivity and remarkably higher PPTs than individuals without pain. ${ }^{12}$ Giesbrecht et al also found a reduction in the PPTs of lumbar paravertebral muscles in people with LBP, as well in some points unrelated to the lower back. ${ }^{13}$ The reduction in PPT 
indicates possible central sensitization and poor adaptive pain in the process of chronic pain, and patients with chronic pain may overemphasize pain by reporting high levels of physical symptoms. ${ }^{14}$

In a comparison of the PPTs between LBP patients and healthy people, the mean PPTs of the erector spinal muscle and gluteus maximus were remarkably lower in the LBP patients, and an abnormally high difference was found at the third lumbar level $\left(2.7 \mathrm{~kg} / \mathrm{cm}^{2}\right) .{ }^{11}$ According to Fischer's definition, a difference more than $2 \mathrm{~kg} / \mathrm{cm}^{2}$ in the value of PPT is considered pathological; hence, PPTs can be used as an indicator to assess muscle sensitivity. ${ }^{15}$ Muscle weakness, fatigue, and reduced activation rates in patients with LBP may contribute to the differences in PPTs ${ }^{16,17}$ Neurobiological or psychosocial factors may lead to lower PPTs in patients with chronic pain. ${ }^{18}$ Therefore, these factors need to be taken into account when interpreting mechanical pain differences.

Various exercises have been recommended to mitigate chronic pain, and different types of exercise produce different analgesic effects. ${ }^{19-23}$ A quasi-experiment found that healthy women experienced an increase in PPTs after completing knee extension strength training, and the effect returned to baseline levels after 20-minute exercise even if the test sites were far from the area of active muscles. ${ }^{24}$ Jones et al recruited 16 healthy adults to complete 20 minutes of moderateintensity aerobic exercise on a cycle ergometer, and the results showed a substantial increase in PPTs but not in heat pain thresholds after exercise. ${ }^{25}$ Tai Chi Quan (TCQ) is a light-to-moderate-intensity exercise with a metabolic equivalent between 2.5 and $6.5 .^{26}$ Known as a mind-body exercise, TCQ has shown beneficial effects on pain intensity and functional disability scores in people with LBP. ${ }^{27}$ However, whether TCQ can improve the PPTs of lower back muscles remains unknown. Considering the factors mentioned above, such as gender, weight, and chronic pain, it is necessary to first know the efficacy of TCQ on PPTs in healthy people. Therefore, this study was to observe the effect of TCQ on the PPTs of lower back muscles in healthy women and to explore the potential changes of PPTs in the subgroup analyses of different weight and psychological statuses. The study may provide preliminary evidence that TCQ can improve PPTs, and also complement the application of TCQ in the field of pain management.

\section{Materials and Methods}

\section{Study Design}

This study was a randomized controlled trial in healthy women. All included participants were randomly assigned to Tai Chi group or control group. The duration of the intervention was 40 minutes, and the assessment time points were baseline and immediately after the intervention. G*Power software was used to calculate sample size with an alpha of 0.05 , a power of 0.80 and an effect size of $0.65 .^{9}$ Thirty participants were included in each group. The study setting is located in the Center of Sports Medicine and Rehabilitation, Shanghai Shangti Orthopaedic Hospital, Shanghai, China.

\section{Randomization and Blinding}

All participants were firstly assigned integer numbers from 1 depending on when they were enrolled. Then, the research assistant obtained a random number table generated by Microsoft Excel 2016 and assigned random numbers to the numbered participant in the same direction. Divide the random number by 2 , and participants with a remainder of 0 were assigned to the Tai Chi group, and those with a remainder of 1 were assigned to the control group. In order to achieve 1:1 allocation, the assistant would continue to select random numbers for remainder adjustment if necessary. The assistant who performed the randomization did not participate in the formal experiment process. Pre- and post-intervention evaluations were performed by the same researcher who were blinded to the group allocation to reduce errors. The participants were not allowed to talk any information about grouping or intervention with the tester. The participants also did not know the purpose of the study to exclude the potential effects of psychological factors on PPTs.

\section{Participants}

All potential participants were recruited from Yangpu District of Shanghai City. Sixty healthy women finally participated in this study. Thirty participants were assigned to practice TCQ for 40 minutes, and the other 30 participants performed 5 
minutes of sham TCQ exercise and 35 minutes of relaxation. Each participant signed a written informed consent before baseline assessments.

The inclusion criteria were as follows: (a) healthy women aged 18-40 years who have no any history of chronic medical conditions; (b) no musculoskeletal pain in the lower back or other areas that occurred in the past month before baseline assessments; (c) no opioids, antidepressants, anti-anxiety medications, or sleeping medications were used in the past month; (d) no non-steroidal anti-inflammatory drugs, shock wave, electrotherapy, or other physiotherapies were used in the last 48 hours before the study; (e) have learned TCQ and have not practiced TCQ regularly in the past 3 months; (f) no exercise habits; (g) no strenuous exercise and no physical fatigue 24 hours before baseline assessments.

The exclusion criteria were as follows: (a) motor and sensory impairments; (b) skin damage or skin disease; (c) had a history of back pain or lumbar surgery; (d) pregnant or preparing to become pregnant; (e) had a history of drug or alcohol abuse; (f) consumption of coffee, alcohol, or smoking within 24 hours prior to assessment.

\section{Interventions}

The movement of TCQ is characterized by moving the limbs with the waist. The intervention program in the Tai Chi group comprises the following movements: (1) Part the Wild Horse's Mane; (2) Pull, Block and Pound; (3) Fair Lady Works at Shuttles; (4) Brush Knee and Twist Step; (5) Golden Rooster Stands on One Leg; (6) Step Back and Whirl Arms on Both Sides; (7) Grasp the Peacock's Tail and (8) Cloudy Hands (Figure 1 Eight Tai Chi Quan movements). Figure S1 provides the brief description of the eight TCQ movements. The eight movements fully embody this characteristic, with changes in the center of gravity in different directions. Each participant in the Tai Chi group was

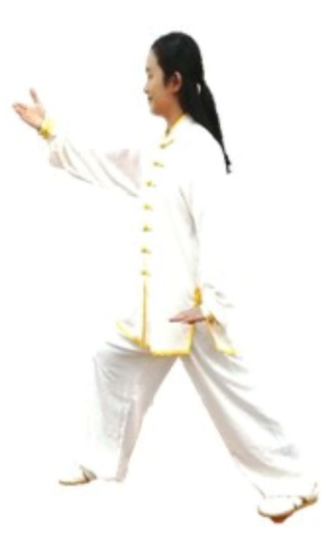

(1) Part the Wild Horse's Mane

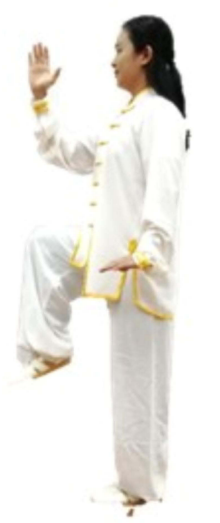

(5) Golden Rooster Stands on One Leg

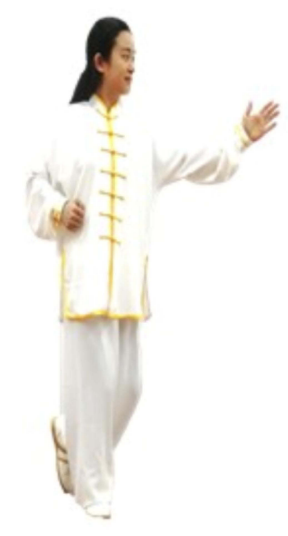

(2) Pull, Block and Pound

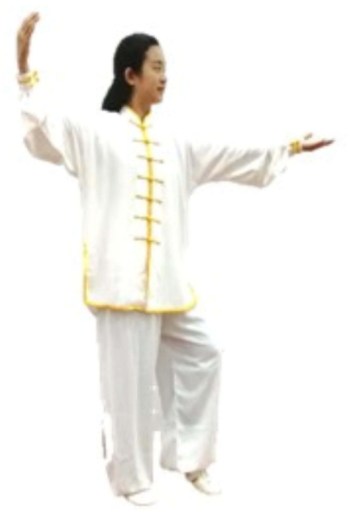

(6) Step Back and Whirl Arms on Both Sides

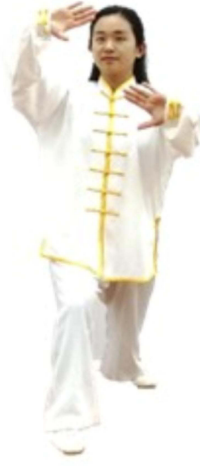

(3) Fair Lady Works at Shuttles

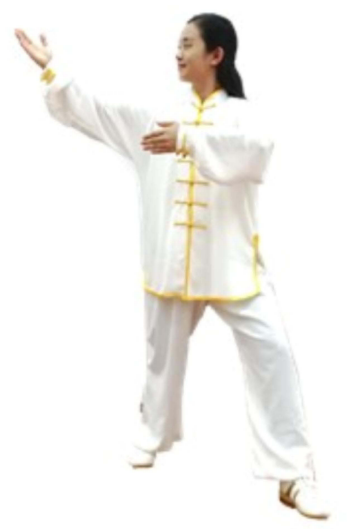

(7) Grasp the Peacock's Tail

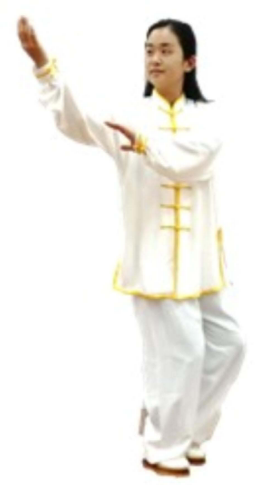

(4) Brush Knee and Twist Step

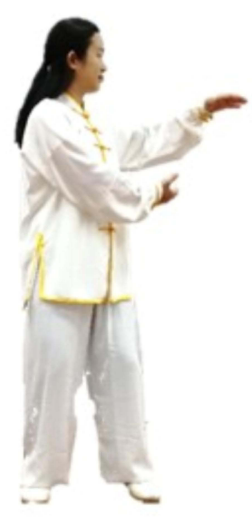

(8) Cloudy Hands

Figure I Eight Tai Chi Quan movements. 
required to complete a 40-minute exercise with one-on-one professional guidance. The participants in the control group practiced sham TCQ for 5 minutes and then rested for 35 minutes in sitting position. Sham TCQ refers to a practice of single movement without any guidance of thought or breathing.

\section{PPT Test}

A hand-held digital manometer (FDX 25 Digital Force Gage, $100 \times 0.1 \mathrm{~N}$ ) was used to evaluate pain threshold. This device has a $1 \mathrm{~cm}^{2}$ rubber probe for contact with the participant's skin. The researcher applied pressure gradually at a rate of $1 \mathrm{~kg}$ per second parallel to the maximum cross-section of the target muscle. The researcher performed three consecutive measurements at the selected test point with an interval of 10 seconds as recovery time, and the average of the three measurements was recorded as the PPT of the test point. The test points of this study were as follows: (1) longissimus thoracis (LT), which is located $1.5 \mathrm{~cm}$ beside the spinous process of the first lumbar spine; (2) iliocostalis lumborum (IL), which is located $3 \mathrm{~cm}$ beside the spinous process of the first lumbar spine; (3) multifidus muscle (M), which is found $1.5 \mathrm{~cm}$ beside the spinous process of the fourth lumbar spine; (4) quadratus lumborum (QL), which is 45 degrees oblique to the midpoint of the line between the lower ribs and the iliac crest in lateral decubitus position; (5) gluteus medius (GM), which is $6 \mathrm{~cm}$ below the posterior superior iliac spine and extends horizontally to the second sacral foramen; (6) supraspinous ligament (SL), which is located midpoint of the line between the fourth and the fifth lumbar spinous processes. The test was performed on the left and right sides, and each participant was assessed in the same order. Before the formal test, the researcher selected the dominant arm for PPT test so that the participant could be familiar with the test process.

\section{Data Collection}

PPT is defined as the amount of pressure at which the participant felt the pressure change to pain. The unit was expressed in kilogram per square centimetre. The PPTs were collected before and after the intervention. Demographic characteristics, including age, body mass index (BMI), degree of education, sitting time, exercise habit, self-rating anxiety scale (SAS) score, general self-efficacy scale score, fear of pain questionnaire (FPQ) score, pain catastrophizing scale (PCS) score and fear-avoidance beliefs questionnaire (FABQ) score, were collected before the intervention.

\section{Statistical Analysis}

Microsoft excel 2016 and IBM SPSS Statistics 20.0 (SPSS Inc, Chicago, USA) were used in data analysis. For the PPTs that satisfy normal distribution, independent sample $t$-test was conducted to compare the difference of the PPTs between the Tai Chi group and the control group, and paired sample $t$-test was conducted to compare PPTs within group. For the PPTs that do not satisfy normal distribution, Mann-Whitney $U$-test was used for intergroup comparison, and Wilcoxon signed-rank test was used for intragroup comparison. Pearson correlation coefficient was used to analyse the correlation between the PPTs and various baseline characteristics. $P<0.05$ was considered statistically significant.

\section{Results}

Sixty-nine potential women were recruited for this study, of which nine were excluded for different reasons (Figure 2 Flow diagram). One person had taken analgesics before the PPT test, seven reported chronic LBP, and one was tolerant to pressure pain. Finally, only 60 participants completed the whole study. The participants in the Tai Chi group and control group had no remarkable differences in demographic characteristics and PPTs at baseline as shown in Tables 1 and 2.

\section{Intragroup Differences in PPTs After Intervention}

The PPT test points with significant differences on the right side in the Tai Chi group were LT ( $p=0.000)$, IL ( $p=0.003)$, $\mathrm{M}(p=0.000)$, QL $(p=0.009), \mathrm{GM}(p=0.000)$ and SL $(p=0.002)$. The test points on the left side also had remarkable differences after intervention as shown in Table 3. Compared with baseline, the PPTs of the right LT and M increased by more than $20 \%$ after the intervention, whereas the PPTs of the right QL and GM increased by $15.4 \%$ and $16.7 \%$, respectively. The PPTs of the left LT, IL, M, QL and GM increased by $15.7 \%, 15.3 \%, 18.9 \%, 19.3 \%$ and $19.1 \%$, respectively. In the control group, the right QL $(p=0.040)$ and the left LT $(p=0.001), \operatorname{IL}(p=0.003)$ and $\mathrm{M}(p=0.026)$ had significant differences after 


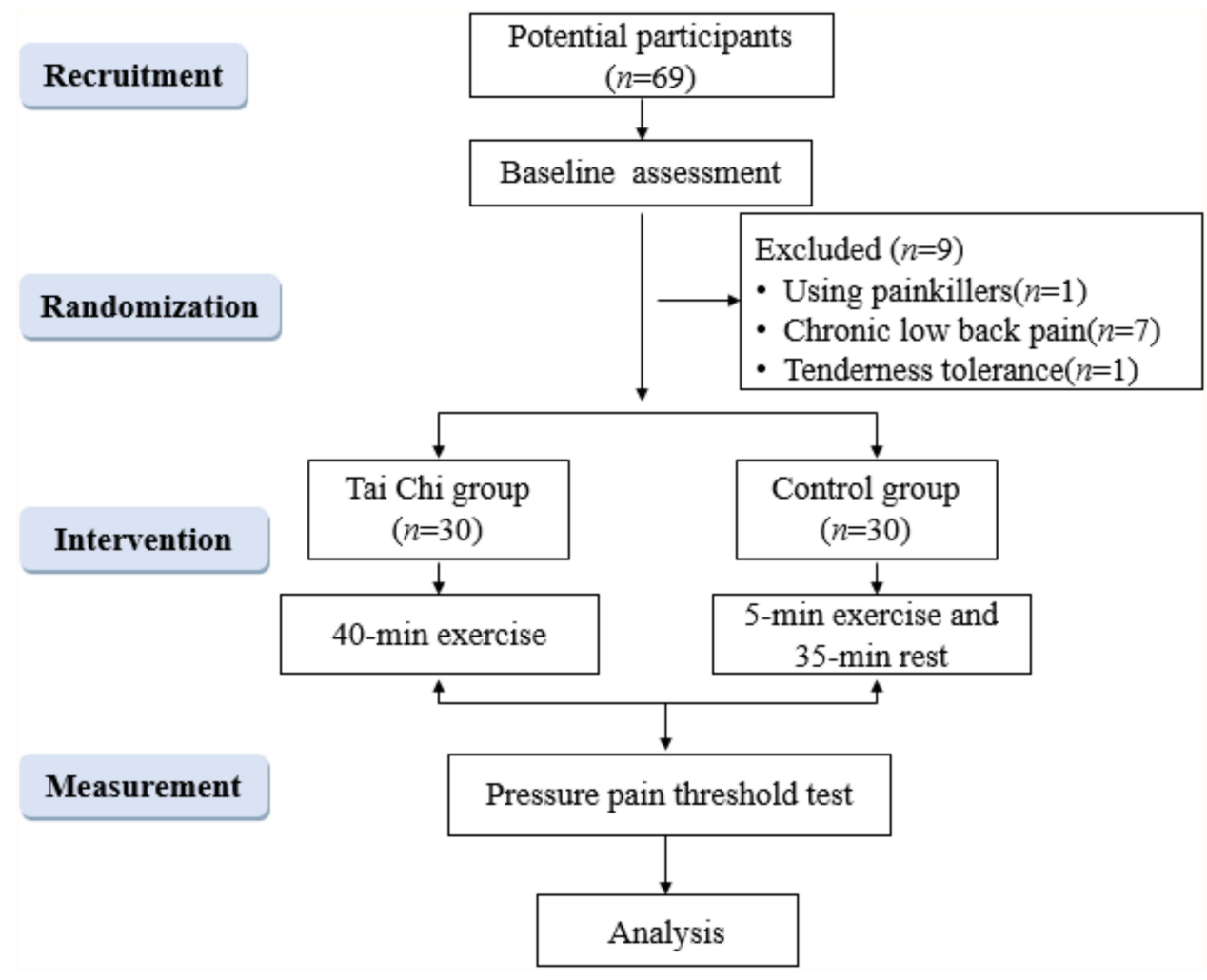

Figure 2 Flow diagram.

intervention. However, the PPTs of the latter three test points decreased. No remarkable difference was observed between the left and right test points in the Tai Chi group and control group at the same level.

\section{Intergroup Differences in PPTs After Intervention}

On the right side, simplified TCQ could effectively increase the PPTs of the LT, IL, M, GM and SL of the Tai Chi group compared with those of the control group (Table 4). On the left side, TCQ also remarkably increased the PPTs of LT, IL, M, QL and GM (Table 4). Overall, 40 minutes of TCQ exercise significantly increased the PPTs of LT $(\mathrm{t}=7.391, p=$ $0.000), \operatorname{IL}(\mathrm{z}=-4.236, p=0.000), \mathrm{M}(\mathrm{z}=-5.411, p=0.000), \mathrm{QL}(\mathrm{z}=-2.506, p=0.012), \mathrm{GM}(\mathrm{t}=5.188, p=0.000)$ and SL $(\mathrm{z}=-3.541, p=0.000)$.

Table I Demographic Characteristics of Participants

\begin{tabular}{|l|l|l|l|}
\hline & Tai Chi Group (n=30) & Control Group (n=30) & p value \\
\hline Age (year) & $23.80 \pm 2.67$ & $23.47 \pm 2.84$ & 0.946 \\
Body mass index $\left(\mathrm{kg} / \mathrm{m}^{2}\right)$ & $20.19 \pm 1.19$ & $19.80 \pm 0.97$ & 0.173 \\
Degree of education & $2.67 \pm 0.61$ & $2.87 \pm 0.63$ & 0.280 \\
Sitting time (hour/day) & $7.50 \pm 2.16$ & $7.80 \pm 1.99$ & 0.756 \\
Exercise time (min/week) ${ }^{\mathrm{b}}$ & $1.53 \pm 0.68$ & $1.23 \pm 0.43$ & 0.072 \\
SAS score & $40.50 \pm 4.30$ & $40.73 \pm 6.88$ & 0.853 \\
GSES score & $26.20 \pm 3.80$ & $26.03 \pm 4.04$ & 0.806 \\
FPQ score & $97.57 \pm 13.73$ & $91.47 \pm 15.97$ & 0.118 \\
PCS score & $17.60 \pm 9.74$ & $16.30 \pm 6.99$ & 0.917 \\
FABQ score & $35.73 \pm 14.69$ & $38.97 \pm 14.91$ & 0.222 \\
\hline
\end{tabular}

Notes: ${ }^{a}$ I for high school, 2 for bachelor's degree, 3 for master's degree and 4 for doctor's degree. ${ }^{b} 0-75$ minutes was defined as I, 76-I50 minutes as 2 , I5I-300 minutes as 3 , and more than 300 minutes as 4 .

Abbreviations: SAS, self-rating anxiety scale; GSES, general self-efficacy scale; FPQ, fear of pain questionnaire; PCS, pain catastrophizing scale; FABQ, fear-avoidance beliefs questionnaire. 
Table 2 Comparison of the Pressure Pain Thresholds Between the Tai Chi Group and the Control Group at Baseline

\begin{tabular}{|c|c|c|c|c|c|c|}
\hline & \multicolumn{2}{|c|}{ Right } & \multirow[t]{2}{*}{$P$ value } & \multicolumn{2}{|c|}{ Left } & \multirow[t]{2}{*}{$P$ value } \\
\hline & $\begin{array}{l}\text { Tai Chi Group } \\
(n=30)\end{array}$ & $\begin{array}{l}\text { Control Group } \\
\qquad(n=30)\end{array}$ & & $\begin{array}{c}\text { Tai Chi Group } \\
(n=30)\end{array}$ & $\begin{array}{l}\text { Control Group } \\
\qquad(n=30)\end{array}$ & \\
\hline Longissimus Thoracis & $4.15 \pm 0.96$ & $3.86 \pm 0.96$ & 0.115 & $4.47 \pm 1.14$ & $4.1 I \pm I .4 I$ & 0.152 \\
\hline Iliocostalis Lumborum & $3.66 \pm 1.05$ & $3.28 \pm 0.77$ & 0.115 & $3.78 \pm 0.94$ & $3.70 \pm 1.15$ & 0.478 \\
\hline Multifidus Muscle & $4.66 \pm 1.19$ & $4.23 \pm 1.46$ & 0.076 & $4.64 \pm 1.57$ & $4.34 \pm 1.42$ & 0.435 \\
\hline Quadratus Lumborum & $1.93 \pm 0.48$ & $I .85 \pm 0.4 \mid$ & 0.504 & $2.11 \pm 0.77$ & $1.84 \pm 0.62$ & 0.258 \\
\hline Gluteus Medius & $3.55 \pm 0.70$ & $3.26 \pm 0.68$ & 0.104 & $3.35 \pm 1.00$ & $2.92 \pm 0.90$ & 0.122 \\
\hline Supraspinous Ligament & $4.44 \pm 1.75$ & $4.22 \pm 1.26$ & 0.779 & - & - & - \\
\hline
\end{tabular}

Table 3 Intra-Group Differences in the Right and Left Pressure Pain Thresholds After Intervention

\begin{tabular}{|l|l|l|l|l|}
\hline & \multicolumn{2}{|c|}{ Tai Chi Group (n=30) } & \multicolumn{2}{c|}{ Control Group (n=30) } \\
\hline & $t$ value & $p$ value & $t$ value & $p$ value \\
Longissimus Thoracis (R) & 5.750 & 0.000 & -1.278 & 0.212 \\
Longissimus Thoracis (L) & 5.178 & 0.000 & -3.900 & 0.001 \\
Iliocostalis Lumborum (R) & 3.181 & 0.003 & -0.942 & 0.354 \\
Iliocostalis Lumborum (L) & 3.966 & 0.000 & -3.267 & 0.003 \\
Multifidus Muscle (R) & 6.196 & 0.000 & -1.363 & 0.183 \\
Multifidus Muscle (L) & 5.427 & 0.000 & -2.355 & 0.026 \\
Quadratus Lumborum (R) & 2.808 & 0.009 & 2.145 & 0.040 \\
Quadratus Lumborum (L) & 3.645 & 0.001 & -0.139 & 0.891 \\
Gluteus Medius (R) & 5.680 & 0.000 & 1.243 & 0.224 \\
Gluteus Medius (L) & 6.459 & 0.000 & -0.464 & 0.646 \\
Supraspinous Ligament & 3.344 & 0.002 & -1.524 & 0.138 \\
\hline
\end{tabular}

Abbreviations: R, right; L, left.

Table 4 Inter-Group Differences in the Right and Left Pressure Pain Thresholds After Intervention

\begin{tabular}{|l|l|l|l|l|l|}
\hline & Tai Chi Group (n=30) & Control Group (n=30) & t/z value & Effect Size & $\boldsymbol{p}$ value \\
\hline Longissimus Thoracis (R) & $0.90 \pm 0.86$ & $-0.14 \pm 0.62$ & 5.416 & 0.570 & 0.000 \\
Longissimus Thoracis (L) & $0.70 \pm 0.74$ & $-0.43 \pm 0.61$ & 6.477 & 0.640 & 0.000 \\
Iliocostalis Lumborum (R) & $0.46 \pm 0.80$ & $-0.10 \pm 0.56$ & 3.141 & 0.376 & 0.003 \\
Iliocostalis Lumborum (L) & $0.58 \pm 0.80$ & $-0.39 \pm 0.66$ & $-4.776^{\mathrm{a}}$ & 0.552 & 0.598 \\
Multifidus Muscle (R) & $0.99 \pm 0.88$ & $-0.16 \pm 0.64$ & $-5.005^{\mathrm{a}}$ & 0.000 \\
Multifidus Muscle (L) & $0.88 \pm 0.89$ & $-0.28 \pm 0.66$ & $5.087^{\mathrm{a}}$ & 0.595 & 0.151 \\
Quadratus Lumborum (R) & $0.30 \pm 0.58$ & $0.15 \pm 0.38$ & $-0.658^{\mathrm{a}}$ & 0.000 \\
Quadratus Lumborum (L) & $0.41 \pm 0.61$ & $-0.01 \pm 0.37$ & $-3.365^{\mathrm{a}}$ & 0.384 & 0.511 \\
Gluteus Medius (R) & $0.59 \pm 0.57$ & $0.08 \pm 0.37$ & 4.080 & 0.469 & 0.001 \\
Gluteus Medius (L) & $0.64 \pm 0.54$ & $-0.05 \pm 0.57$ & 4.790 & 0.528 & 0.000 \\
Supraspinous Ligament & $0.61 \pm 1.00$ & $-0.23 \pm 0.8 \mathrm{O}$ & $-3.54 \mathrm{I}^{\mathrm{a}}$ & 0.419 & 0.000 \\
\hline
\end{tabular}

Note: ${ }^{a}$ nonparametric test.

Abbreviations: R, right; L, left.

\section{Correlation Analysis of PPTs and Demographical Characteristics}

PPT had no substantial correlation with BMI, sitting time, FPQ score, PCS score and FABQ score at the six test points. SAS score had a negative correlation with the overall PPT of the $\mathrm{M}(\mathrm{R}=-0.256, p=0.048)$ but not with the PPTs of the other test points. The improvement of the overall PPTs of the IL and M after TCQ exercise was positively correlated with sitting time ( $\mathrm{R}=0.398, p=0.029 ; \mathrm{R}=0.365, p=0.047$ ), and the overall PPT of QL was positively correlated with SAS 
Table 5 Comparison of Pressure Pain Thresholds of Body Mass Index Subgroup in the Tai Chi Group

\begin{tabular}{|l|l|l|l|l|}
\hline & BMI < 2 I & BMI $\geq \mathbf{2 I}$ & t/z value & $\boldsymbol{p}$ value \\
\hline Longissimus Thoracis & $0.66 \pm 0.78$ & $0.94 \pm 0.55$ & $-1.763^{\mathrm{a}}$ & 0.078 \\
Illiocostalis Lumborum & $0.30 \pm 0.46$ & $0.27 \pm 0.35$ & 0.205 & 0.839 \\
Multifidus Muscle & $0.83 \pm 0.92$ & $1.04 \pm 0.66$ & $-1.431^{\mathrm{a}}$ & 0.152 \\
Quadratus Lumborum & $0.13 \pm 0.27$ & $0.58 \pm 0.64$ & $-2.324^{\mathrm{a}}$ & 0.020 \\
Gluteus Medius & $0.49 \pm 0.5 \mathrm{I}$ & $0.74 \pm 0.49$ & $-1.37 \mathrm{I}$ & $0.18 \mathrm{I}$ \\
Supraspinous Ligament & $0.34 \pm 1.1 \mathrm{I}$ & $0.88 \pm 0.83$ & $-2.157^{\mathrm{a}}$ & $0.03 \mathrm{I}$ \\
\hline
\end{tabular}

Note: ${ }^{a}$ Nonparametric test.

Abbreviation: BMI, body mass index.

score $(\mathrm{R}=0.383, p=0.037)$. The results suggested that TCQ may be more effective in improving PPTs in sedentary people and people with anxiety.

\section{Comparison of PPTs Between the Subgroups of Tai Chi Group}

All the PPTs in the Tai Chi group showed an increasing trend. Therefore, exploratory subgroup analyses were conducted. The group with a BMI of 21 or greater had significant improvement in the PPTs of QL $(p=0.020)$ and SL $(p=0.031)$ compared with the group with a BMI less than 21 (Table 5). Within the normal BMI range, the increase in PPTs may be associated with BMI. The group with a FPQ score of 100 or greater had a significant improvement in the PPT of LT ( $p=$ 0.026) compared with the group with a FPQ score less than 100 (Table 6). No remarkable difference was observed at the other test points and in the subgroup analyses of sitting time, SAS score, PCS score and FABQ score.

\section{Discussion}

At present, the assessment of PPTs using pressure algometers is an important part of pain research. This study found that the PPTs of the LT, IL, M, QL, and GM on the left and right sides of healthy women remarkably increased after 40 minutes of TCQ practice, and the PPTs of most of the test points improved by at least $15 \%$. Compared with control group, Tai Chi group showed important improvements in the PPTs of lower back muscles.

Pain thresholds increase during and after exercise because of the release of opioids and growth factors from the central nervous system, as well as the pain inhibition mechanism. ${ }^{28}$ Different forms of exercise may produce different analgesic effects and have different effects on PPTs. Hoffman et al concluded that an exercise intensity of more than $50 \%$ of maximum oxygen uptake and an exercise time of more than 10 minutes achieve the analgesic effect and reduce pressure pain test scores in healthy people. ${ }^{29}$ Paungmali et al found that stabilization exercise induces pain regulation in LBP patients by improving pain thresholds and reducing pain intensity. ${ }^{30}$ Female patients with fibromyalgia showed significant improvements in PPTs, tenderness points, muscle stiffness, flexibility, and other psychological outcomes after TCQ intervention three times a week for 28 weeks. ${ }^{31}$ However, studies on the change in PPTs after immediate TCQ practice are lacking. The present study complements this part and finds that healthy women had higher PPTs in the lower

Table 6 Comparison of Pressure Pain Thresholds of Fear of Pain Questionnaire Score Subgroup in the Tai Chi Group

\begin{tabular}{|l|l|l|l|l|}
\hline & FPQ Score $<100$ & FPQ Score $\geq \mathbf{1 0 0}$ & t/z value & P value \\
\hline Longissimus Thoracis & $0.56 \pm 0.61$ & $1.04 \pm 0.68$ & $-2.219^{\mathrm{a}}$ & 0.026 \\
Iliocostalis Lumborum & $0.32 \pm 0.43$ & $0.26 \pm 0.37$ & 0.450 & 0.656 \\
Multifidus Muscle & $0.78 \pm 0.64$ & $1.09 \pm 0.92$ & $-0.892^{\mathrm{a}}$ & $-0.788^{\mathrm{a}}$ \\
Quadratus Lumborum & $0.23 \pm 0.38$ & $0.47 \pm 0.65$ & $-0.684^{\mathrm{a}}$ & 0.373 \\
Gluteus Medius & $0.52 \pm 0.52$ & $0.72 \pm 0.49$ & $-1.348^{\mathrm{a}}$ & 0.494 \\
Supraspinous Ligament & $0.37 \pm 0.78$ & $0.86 \pm 1.16$ & 0.188 \\
\hline
\end{tabular}

Note: ${ }^{a}$ Nonparametric test.

Abbreviation: $\mathrm{FPQ}$, fear of pain questionnaire. 
back muscles and gluteus medium following 40 minutes of TCQ practice. This result was consistent with the results of the above studies and shows that TCQ can relieve pain, reduce mechanical pressure sensitivity and improve PPTs.

TCQ is an aerobic exercise suitable for people of all ages. TCQ is widely used in the field of chronic diseases, such as knee osteoarthritis, headache, LBP, and fibromyalgia. ${ }^{32-34}$ Meta-analyses showed that sufficient TCQ has an immediate effect on chronic LBP and TCQ is more effective in the short-term improvement of pain and disability than no treatment or routine care. ${ }^{33}$ Therefore, TCQ has the potential of improving chronic pain conditions.

TCQ combines thought, breath, and power. Thought refers to a high degree of attention, and then the practitioner can easily weak their attention to pain during the practice. ${ }^{35}$ The participants may have better self-management in the face of mechanical pressure stimuli after mindful-guided exercise. TCQ practiced in groups helps relieve interpersonal stress, thereby reducing subjective pain perception. ${ }^{36}$ At the same time, breathing coordination helps to relieve mood and has a beneficial effect on self-efficacy and quality of life. ${ }^{37}$ The eight movements of the TCQ intervention in this study emphasised the process of lumbosacral-pelvic rotation and the transformation of the centre of gravity in all directions. These movements effectively stimulated the lower back muscles and improved the muscle strength and stability of the trunk. Long-term regular TCQ practice can improve the neuromuscular reaction of the trunk, hip, and lower extremity muscles. ${ }^{38}$ Also, the blood flow of the trunk and lower extremity muscles increases during exercise, which may promote the metabolism of inflammatory products and thus improve the pain threshold. ${ }^{39}$ Unlike daily physical activities, TCQ involves more brain activity. The functional homogeneity of the left anterior cingulate cortex decreased and the thickness of the inferior cortex of the insular ring sulcus increased in those who practiced TCQ for a long time. ${ }^{40}$ Changes in these brain regions may contribute to better pain management.

\section{Limitations and Future Direction}

This study was the first to observe the effect of TCQ on the PPTs of lower back muscles in healthy women and was helpful to realise the potential benefit of TCQ in pain management from a new perspective. However, this study has several limitations. Firstly, only healthy women were selected for this study. Men and women have differences in pain perception. Therefore, the next step is to study the effect of TCQ on the PPTs in healthy men. Besides, patients with chronic pain have lower baseline pain thresholds than healthy individuals; thus, the effects of exercise-induced hypoalgesia may also vary. Observing the influence of TCQ on the PPTs in LBP patients will be necessary. Secondly, the interaction between exercise intensity and exercise duration is more likely to influence whether hypoalgesia and low pain sensitivity occur after exercise. ${ }^{41}$ Therefore, future research should pay attention to the influence of different TCQ exercise durations and exercise intensities on PPTs. Thirdly, even though the PPT test was performed by the same researcher, errors were still found in the artificially controlled pressure rates. Fourthly, the PPT is still an unavoidable subjective experience. Future study can explore the neural mechanism of TCQ that affects PPTs.

\section{Conclusion}

Forty minutes of TCQ exercise considerably increased the PPTs of lower back muscles in healthy women compared with the control group, and thresholds at these points increased by an average of $17.2 \%$.

\section{Data Sharing Statement}

The data that support the findings of this study are available from the corresponding author upon reasonable request.

\section{Ethics Approval and Informed Consent}

This study complied with the Declaration of Helsinki and this study was approved by the Ethics Committee of the Shanghai University of Sport (number 102772019RT039), and was registered at the Chinese Clinical Trial Registry (number: ChiCTR2000041272).

\section{Acknowledgment}

SK as one of the co-authors agreed to publish her images presented in the Figure 1. 


\section{Author Contributions}

All authors made a significant contribution to the work reported, whether that is in the conception, study design, execution, acquisition of data, analysis and interpretation, or in all these areas; took part in drafting, revising or critically reviewing the article; gave final approval of the version to be published; have agreed on the journal to which the article has been submitted; and agree to be accountable for all aspects of the work.

\section{Funding}

The study was supported by the Science and Technology Commission of Shanghai Municipality [grant number 19080503100] and the Shanghai Key Lab of Human Performance (Shanghai University of Sport) [grant number 11DZ2261100].

\section{Disclosure}

The authors declare that they have no conflicts of interest for this work.

\section{References}

1. Gupta A, McBeth J, Macfarlane GJ, et al. Pressure pain thresholds and tender point counts as predictors of new chronic widespread pain in somatising subjects. Ann Rheum Dis. 2007;66(4):517-521. doi:10.1136/ard.2006.054650

2. Ji HM, Kim HJ, Han SJ. Extracorporeal shock wave therapy in myofascial pain syndrome of upper trapezius. Ann Rehabil Med. 2012;36 (5):675-680. doi:10.5535/arm.2012.36.5.675

3. Zheng Z, Wang J, Gao Q, et al. Therapeutic evaluation of lumbar tender point deep massage for chronic non-specific low back pain. $J$ Tradit Chin Med. 2012;32(4):534-537. doi:10.1016/s0254-6272(13)60066-7

4. Melia M, Geissler B, König J, et al. Pressure pain thresholds: subject factors and the meaning of peak pressures. Eur J Pain. 2019;23(1):167-182. doi:10.1002/ejp.1298

5. Waller R, Smith AJ, O'Sullivan PB, et al. Pressure and cold pain threshold reference values in a large, young adult, pain-free population. Scand J Pain. 2016;13:114-122. doi:10.1016/j.sjpain.2016.08.003

6. Khimich S. Level of sensitivity of pain in patients with obesity. Acta Chir Hung. 1997;36(1-4):166-167.

7. Amiri M, Alavinia M, Singh M, Kumbhare D. Pressure pain threshold in patients with chronic pain: a systematic review and meta-analysis. Am J Phys Med Rehabil. 2021;100:656-674. doi:10.1097/PHM.0000000000001603

8. Hartvigsen J, Hancock MJ, Kongsted A, et al. What low back pain is and why we need to pay attention. Lancet. 2018;391(10137):2356-2367. doi:10.1016/S0140-6736(18)30480-X

9. Nicholas M, Vlaeyen JWS, Rief W, et al. The IASP classification of chronic pain for ICD-11: chronic primary pain. Pain. 2019;160(1):28-37. doi:10.1097/j.pain.0000000000001390

10. Imamura M, Alfieri FM, Filippo TR, Battistella LR. Pressure pain thresholds in patients with chronic nonspecific low back pain. $J$ Back Musculoskelet Rehabil. 2016;29(2):327-336. doi:10.3233/BMR-150636

11. Farasyn A, Meeusen R. The influence of non-specific low back pain on pressure pain thresholds and disability. Eur J Pain. 2005;9(4):375-381. doi:10.1016/j.ejpain.2004.09.005

12. Peters ML, Schmidt AJ. Differences in pain perception and sensory discrimination between chronic low back pain patients and healthy controls. J Psychosom Res. 1992;36(1):47-53. doi:10.1016/0022-3999(92)90113-G

13. Giesbrecht RJ, Battié MC. A comparison of pressure pain detection thresholds in people with chronic low back pain and volunteers without pain. Phys Ther. 2005;85(10):1085-1092. doi:10.1093/ptj/85.10.1085

14. Ji RR, Nackley A, Huh Y, Terrando N, Maixner W. Neuroinflammation and central sensitization in chronic and widespread pain. Anesthesiology. 2018;129(2):343-366. doi:10.1097/ALN.0000000000002130

15. Fischer AA. Pressure algometry over normal muscles. Standard values, validity and reproducibility of pressure threshold. Pain. 1987;30 (1):115-126. doi:10.1016/0304-3959(87)90089-3

16. Zheng YL, Wang XF, Chen BL, et al. Effect of 12-week whole-body vibration exercise on lumbopelvic proprioception and pain control in young adults with nonspecific low back pain. Med Sci Monit. 2019;25:443-452. doi:10.12659/MSM.912047

17. Lee JH, Hoshino Y, Nakamura K, Kariya Y, Saita K, Ito K. Trunk muscle weakness as a risk factor for low back pain. A 5-year prospective study. Spine. 1999;24(1):54-57. doi:10.1097/00007632-199901010-00013

18. Brellenthin AG, Crombie KM, Cook DB, Sehgal N, Koltyn KF. Psychosocial influences on exercise-induced hypoalgesia. Pain Med. 2017;18 (3):538-550. doi: $10.1093 / \mathrm{pm} / \mathrm{pnw} 275$

19. Rice D, Nijs J, Kosek E, et al. Exercise-induced hypoalgesia in pain-free and chronic pain populations: state of the art and future directions. $J$ Pain. 2019;20(11):1249-1266. doi:10.1016/j.jpain.2019.03.005

20. Koltyn KF, Brellenthin AG, Cook DB, Sehgal N, Hillard C. Mechanisms of exercise-induced hypoalgesia. J Pain. 2014;15(12):1294-1304. doi:10.1016/j.jpain.2014.09.006

21. Zheng K, Chen C, Yang S, Wang X. Aerobic exercise attenuates pain sensitivity: an event-related potential study. Front Neurosci. 2021;15:735470. doi: $10.3389 /$ fnins. 2021.735470

22. Wu B, Zhou L, Chen C, Wang J, Hu LI, Wang X. Effects of exercise-induced hypoalgesia and its neural mechanisms. Med Sci Sports Exerc. 2022;54(2):220-231. doi:10.1249/MSS.0000000000002781

23. Peng MS, Wang R, Wang YZ, et al. Efficacy of therapeutic aquatic exercise vs physical therapy modalities for patients with chronic low back pain: a randomized clinical trial. JAMA Netw Open. 2022;5(1):e2142069. doi:10.1001/jamanetworkopen.2021.42069 
24. Poli-Neto OB, Oliveira AMZ, Salata MC, et al. Strength exercise has different effects on pressure pain thresholds in women with endometriosis-related symptoms and healthy controls: a quasi-experimental study. Pain Med. 2020;21(10):2280-2287. doi:10.1093/pm/pnz310

25. Jones MD, Nuzzo JL, Taylor JL, Barry BK. Aerobic exercise reduces pressure more than heat pain sensitivity in healthy adults. Pain Med. 2019;20 (8):1534-1546. doi:10.1093/pm/pny289

26. Peng PW. Tai chi and chronic pain. Reg Anesth Pain Med. 2012;37(4):372-382. doi:10.1097/AAP.0b013e31824f6629

27. Hall AM, Maher CG, Lam P, Ferreira M, Latimer J. Tai chi exercise for treatment of pain and disability in people with persistent low back pain: a randomized controlled trial. Arthritis Care Res. 2011;63(11):1576-1583. doi:10.1002/acr.20594

28. Koltyn KF. Analgesia following exercise: a review. Sports Med. 2000;29(2):85-98. doi:10.2165/00007256-200029020-00002

29. Hoffman MD, Shepanski MA, Ruble SB, Valic Z, Buckwalter JB, Clifford PS. Intensity and duration threshold for aerobic exercise-induced analgesia to pressure pain. Arch Phys Med Rehabil. 2004;85(7):1183-1187. doi:10.1016/j.apmr.2003.09.010

30. Paungmali A, Joseph LH, Sitilertpisan P, Pirunsan U, Uthaikhup S. Lumbopelvic core stabilization exercise and pain modulation among individuals with chronic nonspecific low back pain. Pain Pract. 2017;17(8):1008-1014. doi:10.1111/papr.12552

31. Romero-Zurita A, Carbonell-Baeza A, Aparicio VA, Ruiz JR, Tercedor P, Delgado-Fernández M. Effectiveness of a tai-chi training and detraining on functional capacity, symptomatology and psychological outcomes in women with fibromyalgia. Evid Based Complement Alternat Med. 2012;2012:614196. doi:10.1155/2012/614196

32. Hall A, Copsey B, Richmond H, et al. Effectiveness of Tai Chi for chronic musculoskeletal pain conditions: updated systematic review and meta-analysis. Phys Ther. 2017;97(2):227-238. doi:10.2522/ptj.20160246

33. Kong LJ, Lauche R, Klose P, et al. Tai Chi for chronic pain conditions: a systematic review and meta-analysis of randomized controlled trials. Sci Rep. 2016;6:25325. doi:10.1038/srep25325

34. Wang C, Schmid CH, Iversen MD, et al. Comparative effectiveness of Tai Chi versus physical therapy for knee osteoarthritis: a randomized trial. Ann Intern Med. 2016;165(2):77-86. doi:10.7326/M15-2143

35. Hilton L, Hempel S, Ewing BA, et al. Mindfulness meditation for chronic pain: systematic review and meta-analysis. Ann Behav Med. 2017;51 (2):199-213. doi:10.1007/s12160-016-9844-2

36. Solloway MR, Taylor SL, Shekelle PG, et al. An evidence map of the effect of Tai Chi on health outcomes. Syst Rev. 2016;5(1):126. doi:10.1186/ s13643-016-0300-y

37. Wang F, Lee EK, Wu T, et al. The effects of tai chi on depression, anxiety, and psychological well-being: a systematic review and meta-analysis. Int J Behav Med. 2014;21(4):605-617. doi:10.1007/s12529-013-9351-9

38. Sun W, Zhang C, Song Q, et al. Effect of 1-year regular Tai Chi on neuromuscular reaction in elderly women: a randomized controlled study. Res Sports Med. 2016;24(2):145-156. doi:10.1080/15438627.2015.1126280

39. Bower JE, Irwin MR. Mind-body therapies and control of inflammatory biology: a descriptive review. Brain Behav Immun. 2016;51:1-11. doi:10.1016/j.bbi.2015.06.012

40. Wei GX, Dong HM, Yang Z, Luo J, Zuo XN. Tai Chi Chuan optimizes the functional organization of the intrinsic human brain architecture in older adults. Front Aging Neurosci. 2014;6:74. doi:10.3389/fnagi.2014.00074

41. Koltyn KF. Exercise-induced hypoalgesia and intensity of exercise. Sports Med. 2002;32(8):477-487. doi:10.2165/00007256-200232080-00001

Journal of Pain Research

Dovepress

\section{Publish your work in this journal}

The Journal of Pain Research is an international, peer reviewed, open access, online journal that welcomes laboratory and clinical findings in the fields of pain research and the prevention and management of pain. Original research, reviews, symposium reports, hypothesis formation and commentaries are all considered for publication. The manuscript management system is completely online and includes a very quick and fair peer-review system, which is all easy to use. Visit http://www.dovepress.com/testimonials.php to read real quotes from published authors.

Submit your manuscript here: https://www.dovepress.com/journal-of-pain-research-journal 